\title{
Caracterização mecânica do gesso para revestimento produzido no Polo Gesseiro do Araripe
}

\author{
Mechanical characterisation of gypsum for coating \\ produced at Araripe Gypsum Production Complex
}

\section{Fernanda Cavalcanti Ferreira J osé Getulio Gomes de Sousa Arnaldo Manoel Pereira Carneiro}

\section{Resumo}

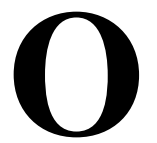

gesso é um material de grande uso na construção civil, destacando-se seu emprego como pasta de gesso para revestimento. No Brasil, o Polo Gesseiro do Araripe é o maior produtor. Percebe-se a necessidade de conhecimento das propriedades e características desse material, principalmente, com foco no produto acabado como revestimento interno em paredes. Nesse contexto, o presente trabalho visa realizar um estudo de caracterização de cinco amostras de gesso do Polo do Araripe, e avaliar a capacidade aderente dos revestimentos executados em pasta desses gessos. $\mathrm{O}$ estudo de caracterização ocorreu no estado anidro, por meio de ensaios de granulometria, massa unitária, massa específica, teores de água livre e de cristalização; no estado fresco, por meio de ensaios de consistência normal, tempo de pega e calorimetria; e no estado endurecido, por ensaios de dureza e resistência à compressão. Os ensaios de resistência de aderência à tração ocorreram nos revestimentos de pasta de gesso executados em base cerâmica, produzidos com e sem pré-umedecimento da base. Com os resultados, verifica-se que as pastas de gesso apresentam desempenhos satisfatórios e semelhantes, independentemente do porte do fabricante; entretanto, alguns requisitos normativos não são atendidos.

Palavras-chave: Gesso para revestimento. Caracterização. Resistência de aderência.

\begin{abstract}
Plaster is a widely used material in the construction industry, mainly as plaster paste for coating. The biggest producer of plaster in Brazil is the Araripe Gypsum Production Complex. There is an evident need to know the properties and characteristics of this material, focusing particularly on the finished product as coating for internal walls. Hence, this research project consisted in a characterisation study of five gypsum samples from the Araripe Complex, and an evaluation of the bond strength of the coatings made of gypsum plaster paste. The characterisation study was carried out in the anhydrous state, by means of granulometry, unit weight, specific gravity, free water and crystallisation content tests; in the fresh state, normal consistency, setting time and calorimetry tests were performed; and in the hardened state, hardness and compressive strength were tested. The bond strength tests were performed on gypsum plaster paste made on a ceramic base, with and without pre-wetting of the base. The results showed that the plaster pastes had satisfactory and similar performances, regardless of the size of the manufacturer, however, some normative requirements were not met.

Keywords: Gypsum plaster. Characterisation. Bond strength.
\end{abstract}

Recebido em 19/04/18 Aceito em 26/11/18

${ }^{3}$ Arnaldo Manoel Pereira Carneiro Universidade Federal de Pernambuco Recife - PE - Brasil 


\section{Introdução}

O gesso é um dos ligantes mais antigos da humanidade. Utiliza-se de uma única matériaprima, o mineral gipsita (sulfato de cálcio dihidratado - $\mathrm{CaSO}_{4} \cdot 2 \mathrm{H}_{2} \mathrm{O}$ ) para sua produção. É por meio da desidratação da gipsita, em função da temperatura e pressão, que se formam as fases: hemi-hidrato $\left(\mathrm{CaSO}_{4} .0,5 \mathrm{H}_{2} \mathrm{O}\right)$, anidrita III $\left(\mathrm{CaSO}_{4} \cdot \mathrm{eH}_{2} \mathrm{O}\right)$, anidrita II $\left(\mathrm{CaSO}_{4}\right)$, anidrita I $\left(\mathrm{CaSO}_{4}\right)$. Esse material apresenta amplo uso na construção civil pelas suas propriedades físicas e mecânicas e baixo custo energético de produção (GARTNER, 2009; JOHN; CINCOTTO, 2007; YU; BROUWERS, 2012), podendo ser utilizado como pasta ou argamassa (gesso + agregado miúdo) (AMERICAN..., 2015; DIAS; CINCOTTO, 1995; JOHN; ANTUNES, 2002). No Brasil, utiliza-se correntemente como pasta (DIAS; CINCOTTO, 1995; JOHN; ANTUNES, 2002; JOHN; CINCOTTO, 2007).

Vários fatores influenciam a qualidade do revestimento de gesso. Tydlitát, Medved e Cerný (2012) explicam que há grande influência da tecnologia de produção de gesso, em particular pelo método de aquecimento e pela temperatura escolhida do processo de calcinação.

Além desses fatores, a relação a/g, a temperatura da água de amassamento, a matéria-prima, a energia de mistura, as impurezas, o tamanho das partículas e a origem mineralógica têm relação com a qualidade do material (ANTUNES; JOHN, 2000; CAMARINI et al., 2016; CAMARINI; PIMENTEL; SÁ, 2011; CAUFIN; PAPO, 1983; CLIFTON, 1973; KARNI; KARNI, 1995; LEWRY; WILLIAMSON, 1994; SCHMITZ; TAVARES, 2009; YE et al., 2011).

O estudo de caracterização e avaliação do gesso para revestimento ocorre nos estados anidro, fresco e endurecido. No estado anidro, faz-se avaliação da granulometria e massa unitária (ABNT, 2017a); e teores de água livre, de cristalização, de óxido de cálcio e de anidrido sulfúrico (ABNT, 2017b). No estado fresco, orienta-se o estudo por meio da consistência e dos tempos de início e fim de pega (ABNT, 2017c). No estado endurecido, por meio da dureza e resistência à compressão (ABNT, 2017d); e resistência de aderência. Essa última avaliação foi realizada por meio de adaptação da NBR 13528 (ABNT, 2010), que é a norma indicada para a avaliação da resistência de aderência à tração de revestimento de argamassas inorgânicas, conforme recomendação da NBR 13207 (ABNT, 2017e). Esses ensaios são fundamentais para conhecimento do material e de sua qualidade, bem como pela possibilidade de utilizá-lo em aplicações de maior valor agregado.
Ensaios de granulometria são de grande importância, pois é uma das formas de classificação do gesso, seja para fins de fundição ou revestimento (ABNT, 2017e). Além disso, essa característica influencia em diversas outras propriedades e características do gesso, como na microestrutura, consistência normal e resistência à compressão (YE et al., 2011). Para Cincotto, Agopyan e Florindo (1988), massa específica e unitária são grandezas que caracterizam a homogeneidade do material.

A determinação do teor de água de cristalização é importante, pois demonstra a condição de calcinação da gipsita, apresentando relação com a composição do gesso, que pode variar entre a gipsita, hemi-hidrato e anidritas. Teores acima de $6,2 \%$ podem indicar a presença de matéria-prima não calcinada e, abaixo de 4,2\%, supercalcinada (JOHN; CINCOTTO, 2007). Outro fator importante é o teor de água livre, que diz respeito à umidade do material, evidenciando as condições de armazenamento.

A consistência normal é um valor de referência para produtores e laboratoristas para avaliação do gesso nos estados fresco e endurecido. No estado fresco, a NBR 13207 (ABNT, 2017e) requisita que o tempo de pega, determinado por meio do aparelho de Vicat, apresente valores mínimos para permitir que se tenha tempo suficiente para manipulação da pasta e execução do revestimento pelo gesseiro. Contudo, essa metodologia apresenta limitações, uma vez que o ensaio foi concebido por analogia ao ensaio empregado para cimento, que possui intervalo de utilização diferente (ANTUNES; JOHN, 2000). Assim sendo, determina-se o tempo de pega por meio de ensaios de calor de hidratação.

Acerca das propriedades no estado endurecido, Dias e Cincotto (1995) afirmam que a importância da determinação da dureza se justifica pela necessidade de se avaliar as qualidades mecânicas do revestimento e a sua capacidade de receber acabamentos como pinturas especiais ou componentes colados.

Quanto ao desempenho do revestimento, é sabido que a aderência é um dos requisitos fundamentais, uma vez que garante bom acabamento e segurança (COSTA; CARASEK, 2009; DIAS; CINCOTTO, 1995). Para Almeida (2014), a rigor, é possível conceber um revestimento com dureza e resistência à compressão satisfatórias, porém que apresentem resistência de aderência à tração questionável, e que, ainda assim, esteja dentro dos parâmetros aceitáveis da normalização brasileira de gesso. De acordo com Dias e Cincotto (1995), a importância dessa avaliação está relacionada a diagnósticos 
mais precisos de deslocamentos verificados na interface base-revestimento, além da possibilidade de estudos em laboratório na busca de melhoramento da aderência do revestimento.

No entanto, durante muito tempo, as normas brasileiras não apresentaram parâmetros específicos para avaliar a qualidade do gesso para revestimento. As avaliações eram limitadas ao gesso como material e não como produto acabado.

De acordo com Barbosa, Ferraz e Santos (2014), o minério do Polo Gesseiro do Araripe é de alta qualidade e de grande abundância, porém suas propriedades não estão sendo exploradas devidamente.

Nesse contexto, a pesquisa visa obter uma ampla caracterização e avaliação de desempenho de cinco gessos para revestimento, produzidos por empresas de diferentes portes no Polo Gesseiro do Araripe; local onde acontece a maior produção desse material no Brasil. Os materiais foram estudados por meio de ensaios de granulometria e módulo de finura, massa unitária, massa específica, teores de água livre e de cristalização, de consistência normal, tempo de pega, calorimetria, dureza e resistência à compressão. Em sequência, foram realizados ensaios de resistência de aderência à tração em revestimentos de pasta de gesso executados em base cerâmica, produzidos com e sem pré-umedecimento da base.

\section{Programa experimental}

O estudo foi dividido em 3 etapas:

(a) obtenção das amostras de gesso;

(b) caracterização das amostras de gesso; e

(c) avaliação dos revestimentos de gesso.

\section{Obtenção das amostras de gesso}

O estudo teve início com a coleta de amostras de 5 marcas de gesso para revestimento (tipo B sem aditivos) fornecidos por diferentes fabricantes na região do Polo Gesseiro do Araripe em Pernambuco, no Brasil. A coleta foi realizada aleatoriamente, sendo as amostras oriundas de fornecedores de diferentes cidades e portes de empresas, distribuídas entre as cidades de Araripina e Ouricuri. O Quadro 1 mostra a codificação dos gessos bem como a cidade de origem.

\section{Caracterização das amostras de gesso}

Foi realizada a caracterização e avaliação dos gessos nos estados anidro, fresco e endurecido por meio dos métodos descritos nos Quadros 2, 3, e 4, respectivamente.

As Figuras 1, 2 e 3 mostram os ensaios nos gessos nos estados anidro, fresco e endurecido, respectivamente.

\section{Avaliação dos revestimentos de gesso}

Após a caracterização dos gessos, foi avaliado o desempenho do gesso como produto acabado por meio da resistência de aderência à tração. A relação água/gesso utilizada para execução dos revestimentos foi de 0,8 , uma vez que é um valor comumente empregado na prática em obras. Utilizou-se a NBR 13528 (ABNT, 2010), que é a norma indicada para a avaliação da resistência de aderência à tração de revestimento de argamassas inorgânicas, uma vez que não existe metodologia para revestimento em pastas de gesso. As pastas de gesso foram aplicadas em bases de blocos cerâmicos, sem chapisco, por ser uma das mais utilizadas no Brasil, considerando ainda as situações com e sem pré-umedecimento da base. A sequência do ensaio é dada na Figura 4.

Quadro 1 - Codificação e origem das amostras de gesso

\begin{tabular}{|c|l|l|l|l|}
\hline $\begin{array}{c}\text { Codificação } \\
\text { do gesso }\end{array}$ & \multicolumn{1}{|c|}{$\begin{array}{c}\text { Cidade de } \\
\text { origem }\end{array}$} & \multicolumn{1}{|c|}{ Porte da empresa } & \multicolumn{1}{|c|}{$\begin{array}{c}\text { Tempo de } \\
\text { produção }\end{array}$} & \multicolumn{1}{|c|}{$\begin{array}{c}\text { Tipo de forno } \\
\text { de calcinação }\end{array}$} \\
\hline G1 & Araripina - PE & - & 1 a 5 anos & - \\
\hline G2 & Ouricuri - PE & - & 11 a 20 anos & Barriga quente \\
\hline G3 & Araripina - PE & 31 a 60 funcionários & 11 a 20 anos & Barriga quente \\
\hline G4 & Araripina - PE & - & 11 a 20 anos & Barriga quente \\
\hline G5 & Araripina - PE & Mais de 100 funcionários & 11 a 20 anos & - \\
\hline
\end{tabular}


Quadro 2 - Caracterização do gesso no estado anidro (pó)

\begin{tabular}{|c|c|}
\hline Característica/propriedade analisada & Método \\
\hline Granulometria & NBR 12127 (ABNT, 2017a) \\
\hline Massa unitária & NBR 12127 (ABNT, 2017a) \\
\hline Massa específica & NM 23 (AMN, 2000) \\
\hline Teores de água livre e água de cristalização & NBR 12130 (ABNT, 2017b) \\
\hline
\end{tabular}

Quadro 3 - Caracterização do gesso no estado fresco (pasta)

\begin{tabular}{|c|c|}
\hline Característica/propriedade analisada & Método \\
\hline Consistência normal & NBR 12128 (ABNT, 2017c) \\
\hline Tempo de pega & NBR 12128 (ABNT, 2017c) \\
\hline Calor de hidratação & Pinheiro (2011) - Calorímetro pseudoadiabático \\
\hline
\end{tabular}

Quadro 4 - Caracterização do gesso no estado endurecido

\begin{tabular}{|c|c|}
\hline Característica/propriedade analisada & Método \\
\hline Dureza & NBR 12129 (ABNT, 2017d) \\
\hline Resistência à compressão & NBR 12129 (ABNT, 2017d) \\
\hline
\end{tabular}

Figura 1 - Ensaios realizados no gesso no estado anidro: a. granulometria; b. massa unitária; c. massa específica

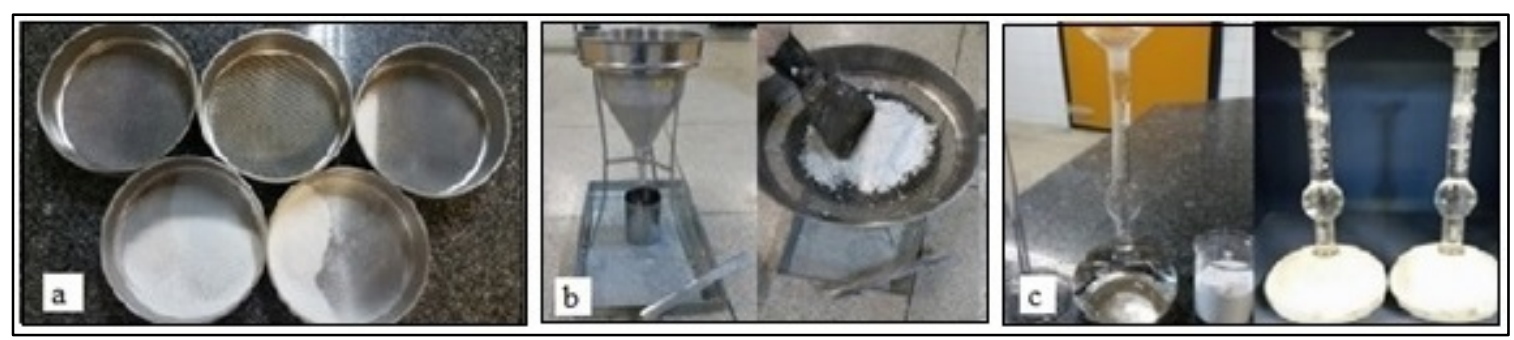

Figura 2 - Ensaios realizados no gesso no estado fresco: (a) tempo de pega; e (b) calor de hidratação

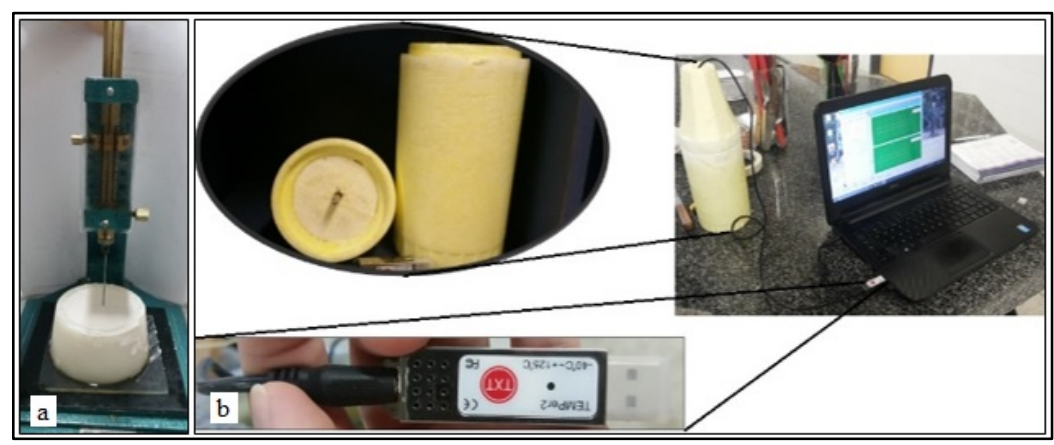

Figura 3 - Ensaios realizados no gesso no estado endurecido: (a) dureza; e (b) resistência à compressão

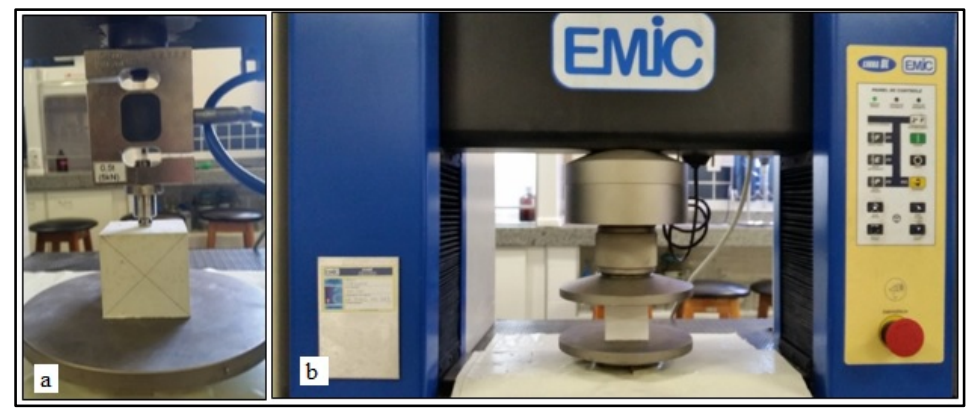


Figura 4 - Sequência do ensaio de resistência de aderência à tração: (a) cortes circulares a seco no revestimento gesso para colagem das pastilhas; (b) pastilhas coladas nos corpos de prova; e (c) e (d) dinamômetro acoplado na pastilha para aplicação de uma força de tração
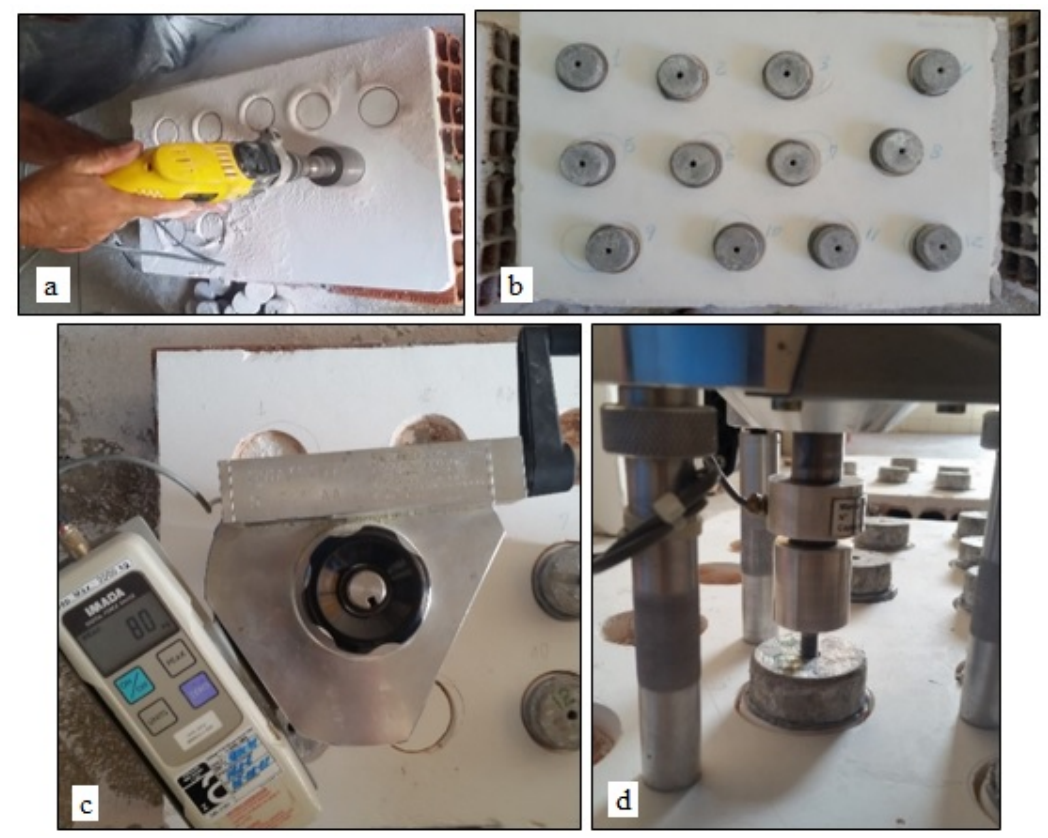

\section{Resultados e discussões}

\section{Caracterização no estado anidro}

\section{Granulometria}

O ensaio de granulometria é de fundamental importância, pois é uma das propriedades que mostra a melhor aplicação para o gesso (COSTA, 2013). Grande parte dos gessos comercializados no Brasil é composta predominantemente de hemihidrato $\beta$, que, de acordo com a granulometria, se classifica como para fundição ou para revestimento (COSTA, 2013).

A Figura 5 apresenta as curvas granulométricas obtidas no ensaio de granulometria.

A partir da soma das porcentagens retidas acumuladas na série de peneiras dividida por 100 , pode-se calcular o módulo de finura desse material. Na Figura 6, observa-se o módulo de finura de cada amostra coletada, com a indicação do desvio padrão no topo de cada barra. Os módulos de finura dos gessos variaram entre 0,59 e 0,72.

A antiga edição da NBR 13207 (ABNT, 1994), que apresentava os requisitos para o gesso de construção civil, classificava o gesso para fundição e para revestimento como sendo fino e grosso em função do módulo de finura. Aquele com um módulo de finura superior a 1,10 era classificado como fino e, do contrário, como grosso.
Entretanto, a recém-lançada edição da mesma norma (ABNT, 2017e) não utiliza o conceito de módulo de finura como um critério de classificação desses materiais, especificando apenas uma granulometria mínima (porcentagem passante) em certa peneira como requisito para sua aplicação na construção civil.

A NBR 13207 (ABNT, 2017e) especifica que os gessos para revestimento tenham, no mínimo, 90\% passante na peneira de abertura de 0,21 mm. Assim, todas as amostras analisadas atendem a esse requisito. A C28/C28M-10 (AMERICAN..., 2015) apresenta o critério de que, no ensaio de granulometria, o gesso para construção para acabametno não deve apresentar material retido na peneira de $1,4 \mathrm{~mm}$ e, pelo menos, $60 \%$ do material deve passar na peneira de $0,150 \mathrm{~mm}$. Todas as amostras estudadas não atendem esse último critério.

Adrien et al. (2016) concluíram em seus estudos que um papel crítico é desempenhado pelo tamanho de partícula (finura) de hemi-hidrato no processo de hidratação e na porosidade do material hidratado.

O módulo de finura dá uma ideia de finura do material, uma vez que representa uma medida da quantidade de material retida nas peneiras. Assim, à medida que aumenta o módulo de finura, tem-se um aumento do tamanho das partículas. Ou seja, para os menores valores de módulo de finura, tem-se um gesso mais fino, com maior área específica e para os maiores valores de módulo de finura, tem-se um gesso mais grosso, com menor área específica. 
Figura 5 - Curvas granulométricas dos gessos

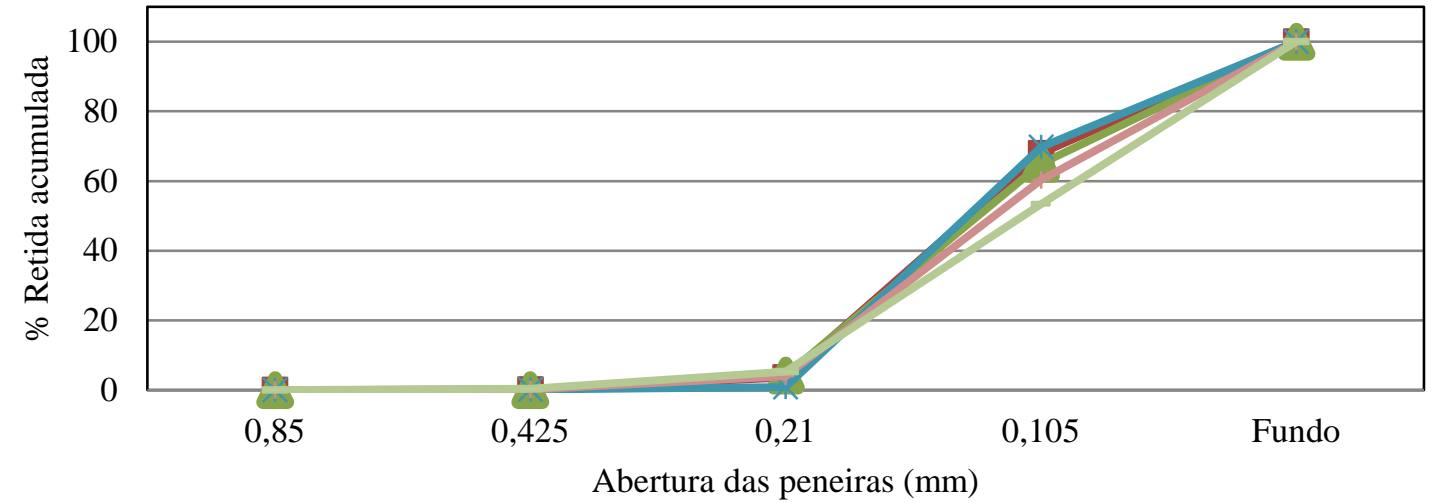

$-\mathrm{G} 1-\mathrm{G} 2 \approx \mathrm{G} 3-\mathrm{G} 4=\mathrm{G} 5$

Figura 6 - Módulo de finura dos gessos

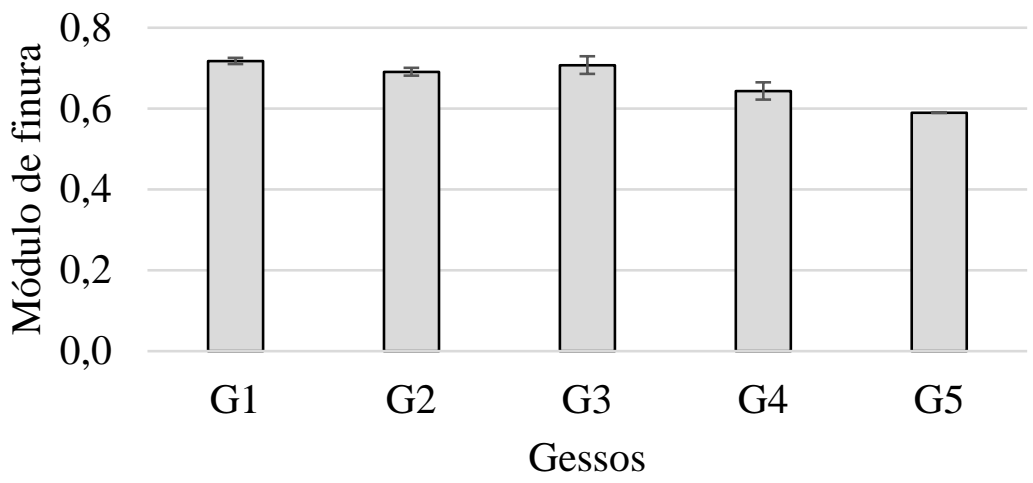

Entretanto, discute-se na literatura (CARNEIRO, 1999) a não representatividade do valor do módulo de finura do agregado, podendo aplicar a mesma ideia na avaliação da granulometria do gesso. Ye et al. (2011) mostraram que, para um gesso $\alpha$, diferentes curvas granulométricas podem gerar os mesmos módulos de finura, e que é possível entender a influência da distribuição do tamanho das partículas nas propriedades do gesso a partir do conjunto distribuição, finura e largura de distribuição.

\section{Massa unitária e massa específica}

A Figura 7 apresenta um gráfico de barras com os valores de massa unitária dos gessos estudados com a barra de erro no topo para indicação do desvio padrão. As massas unitárias variaram entre 0,58 $\mathrm{g} / \mathrm{cm}^{3}$ e $0,70 \mathrm{~g} / \mathrm{cm}^{3}$; valores que se encontram próximos àqueles apresentados na literatura (BARDELLA, 2011; COSTA, 2013; SOUSA; ALMEIDA; ALMEIDA, 2015).

A NBR 13207 (ABNT, 2017e) solicita que o valor mínimo de massa unitária seja 600 kg/m³ $(0,6$ $\left.\mathrm{g} / \mathrm{cm}^{3}\right)$. Logo, somente a amostra G3 é não conforme. Fatores como características do material, condições ambientes e de operação também podem interferir na massa unitária (BARDELLA, 2011).

Os valores obtidos no ensaio de massa específica estão apresentados na Figura 8.

A variação de massa específica foi de $2,54 \mathrm{~g} / \mathrm{cm}^{3}$ a $2,60 \mathrm{~g} / \mathrm{cm}^{3}$, ou seja, uma diferença de até 0,06 $\mathrm{g} / \mathrm{cm}^{3}$. Os valores encontrados estão compatíveis com os obtidos por Cincotto, Agopyan e Florindo (1988) quando os autores analisaram 15 gessos comercializados em São Paulo no ano de 1988.

Essa variação de massa unitária e massa específica podem ser atribuídas ao arranjo dos cristais na massa do material e à estrutura cristalina que depende das condições de produção do hemihidrato (BARDELLA, 2011).

\section{Água livre e de cristalização}

Os resultados dos teores de água livre e de cristalização dos gessos obtidos segundo a NBR 12130 (ABNT, 2017b) se encontram na Tabela 1. 
Figura 7 - Massa unitária dos gessos

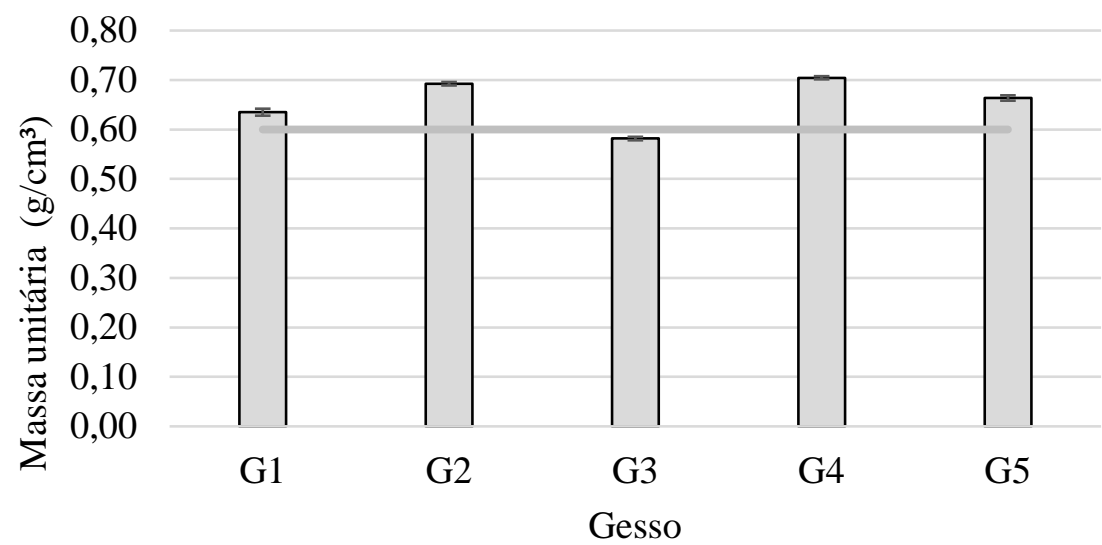

$\square$ Massa unitária $\left(\mathrm{g} / \mathrm{cm}^{3}\right)$

Mínimo NBR 13207 (ABNT, 2017e)

Figura 8 - Massa específica dos gessos
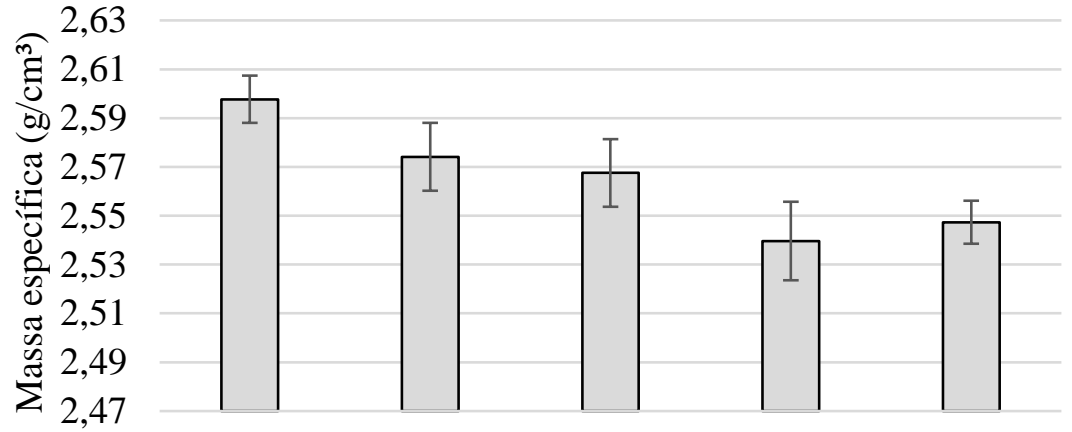

G1

G2

G3

G4

G5

Gesso

Tabela 1 - Água livre e de cristalização dos gessos

\begin{tabular}{|c|c|c|c|c|}
\hline GESSO & $\begin{array}{c}\text { Água livre } \\
(\%)\end{array}$ & $\begin{array}{l}\text { Limite água } \\
\text { livre (\%) - } \\
\text { NBR 13207 } \\
\text { (ABNT, 2017e) }\end{array}$ & $\begin{array}{c}\text { Água de } \\
\text { cristalização (\%) }\end{array}$ & $\begin{array}{c}\text { Limite água de } \\
\text { cristalização (\%) - NBR } \\
13207 \text { (ABNT, 2017e) }\end{array}$ \\
\hline G1 & $0,73 \pm 0,01$ & \multirow{5}{*}{$\leq 1,3 \%$} & $7,1276 \pm 0,0415$ & \multirow{5}{*}{$4,2 \%$ a $6,2 \%$} \\
\hline G2 & 0,54 & & $7,3706 \pm 0,1847$ & \\
\hline G3 & 0,30 & & $6,5299 \pm 0,3231$ & \\
\hline G4 & 0,08 & & $6,8712 \pm 0,1733$ & \\
\hline G5 & $0,56 \pm 0,03$ & & $6,8890 \pm 0,1356$ & \\
\hline
\end{tabular}

A água livre diz respeito à água disponível, não ligada, que está presente entre os grãos e é eliminada com facilidade e depende das condições ambientes. O teor de água livre dos gessos variou em um intervalo de $0,08 \%$ a $0,73 \%$. De acordo com a NBR 13207 (ABNT, 2017e), o gesso para construção civil deve apresentar um máximo de
1,30\% de água livre. Sendo assim, todas as amostras estudadas atendem a esse critério.

A água de cristalização é a água que participa da estrutura cristalina do material. Verifica-se que esse valor variou entre $6,5 \%$ e $7,4 \%$. Conforme a NBR 13207 (ABNT, 2017e), o gesso deve apresentar um teor entre $4,2 \%$ a $6,2 \%$; logo, todas as amostras ultrapassam o limite normativo. 
John e Cincotto (2007) propuseram uma hipótese simplificada para determinar os compostos constituintes do gesso (gipsita, hemi-hidrato e anidrita) com base no teor de água combinada, conforme mostra o Quadro 5. Assim, estima-se que todas as amostras estudadas apresentam gipsita e hemi-hidrato.

\section{Caracterização no estado fresco}

\section{Consistência normal}

A NBR 12128 (ABNT, 2017e) define a consistência normal como o valor da relação água/gesso que se obtém uma fluidez da pasta de gesso adequada para a manipulação. A mesma norma descreve o método de ensaio para determinação do seu valor como aquele que se obtém uma penetração de $30 \mathrm{~mm}$ do aparelho de Vicat modificado. A Tabela 2 mostra a consistência normal dos gessos. Os valores encontrados estão numa faixa de relação água/gesso entre 0,44 e 0,48 , faixa de valores encontrados por pesquisadores na literatura.

A consistência normal é um valor de referência para avaliação do gesso em laboratórios, uma vez que, na prática, não haveria possibilidade de aplicação de um revestimento com essa relação, exceto com a utilização de aditivos, dadas as condições de trabalhabilidade e tempo de pega reduzidos.

Karni e Karni (1995) explicam que a quantidade de água necessária para hidratação desse material é 17\% em massa; no entanto, é necessário muito mais que isso para atingir a consistência plástica desejada, sendo $80 \%$ um valor usual para execução de revestimentos.

\section{Tempos de pega}

Os tempos de início e fim de pega para as pastas estão apresentados na Figura 9.

De acordo com a NBR 13207 (ABNT, 2017e), uma pasta de gesso para revestimento sem aditivo dosada para consistência normal deve apresentar um tempo de início e fim de pega de, no mínimo, 10 min e 35 min, respectivamente. Os tempos de início de pega obtidos se encontram entre 10 min e $18 \mathrm{~min}$; logo, todas as amostras estão em conformidade. Quanto ao tempo de fim de pega, os valores encontrados foram entre 13 min e 25 min; portanto, nenhuma amostra atende a esse critério.

Os tempos de início e fim de pega dos gessos estudados foram semelhantes aos encontrados por autores na literatura, como Antunes (1999), Cincotto, Agopyan e Florindo (1988) e De Milito (2001).

As diferenças ocorridas nos tempos de pega são devido a diferenças da matéria-prima, ao processo de calcinação, ao tamanho das partículas e à relação água/gesso. John e Cincotto (2007) e Cardoso et al. (2009) acrescentam a influência do procedimento de mistura na pega e no endurecimento de amostras provenientes da mesma matéria-prima.

No início de pega determinado pela agulha de Vicat, pelo método da NBR 12128 (ABNT, 2017c), aproximadamente $10 \%$ de di-hidrato está formado (STAV; BENTUR, $1995{ }^{1}$ apud ANTUNES; JOHN, 2000). Para o fim da pega determinado pela agulha de Vicat, a relação é a inversa, isto é, no instante em que ocorre o fim da pega a pasta está excessivamente rígida, não sendo mais possível a sua utilização (ANTUNES; JOHN, 2000).

\section{Quadro 5 - Hipótese simplificada para determinação dos compostos constituintes do gesso por meio do} teor de água de cristalização

\begin{tabular}{|c|c|}
\hline $\mathbf{H}_{2} \mathrm{O}$ combinada - água de cristalização & Compostos constituintes do gesso \\
\hline Se $\mathrm{H}_{2} \mathrm{O}<6,2 \%$ & Há anidrita e hemi-hidrato \\
\hline Se $\mathrm{H}_{2} \mathrm{O}>6,2 \%$ & Há gipsita e hemi-hidrato \\
\hline Se $\mathrm{H}_{2} \mathrm{O}=6,2 \%$ & Há somente hemi-hidrato \\
\hline
\end{tabular}

Fonte: J ohn e Cincotto (2007).

Tabela 2 - Consistência normal dos gessos

\begin{tabular}{c|c}
\hline Gesso & Relação a/g da consistência normal \\
\hline G1 & 0,48 \\
G2 & 0,45 \\
G3 & 0,47 \\
G4 & 0,44 \\
G5 & 0,47 \\
\hline
\end{tabular}

ISTAV, E.; BENTUR, A. Characterization of the Hydration Process of Calcium Sulfate Hemihydrate by Simultaneous Evaluations of
Chemical and Physical Parameters. Advances in Cement Research, v. 7, n. 27, p. 113-116, 1995. 
Figura 9 - Início e fim de pega das pastas com consistência normal

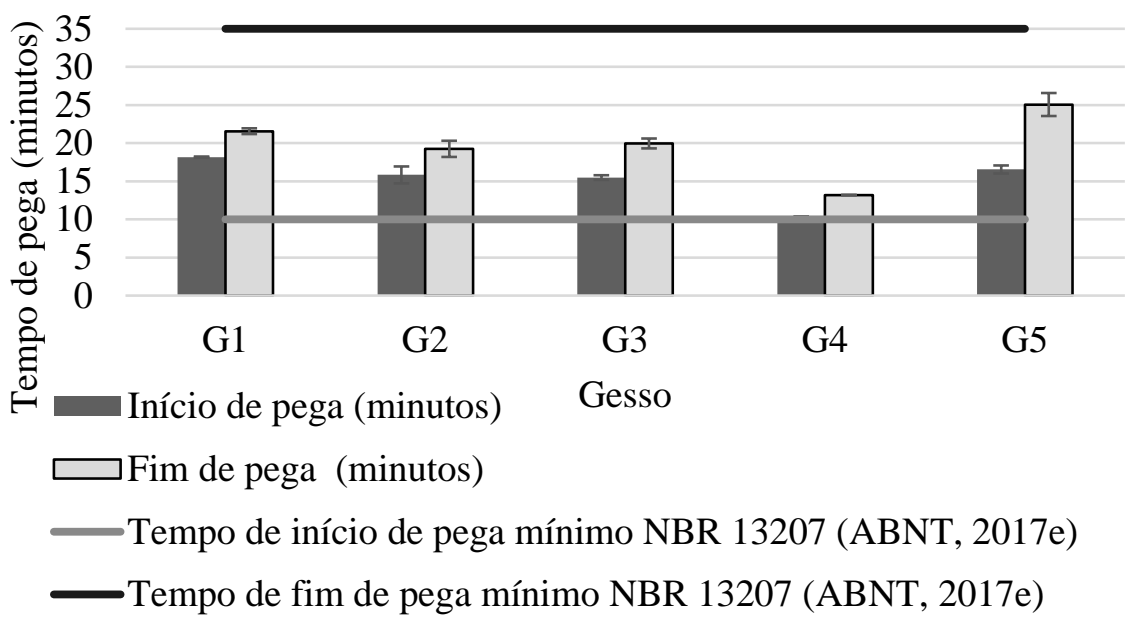

Assim, os tempos de início e fim de pega determinados pela NBR 12128 (ABNT, 2017c) são de pouca utilidade para o construtor, unicamente aplicável para controle do processo de produção do gesso, pois, na prática, o gesseiro inicia a execução do revestimento antes do início de pega e termina antes do fim de pega.

Além disso, em canteiro de obras, a temperatura ambiente vai ter influência nos tempos de início e fim de pega durante a execução dos revestimentos. Os resultados obtidos foram em temperatura ambiente controlada, aproximadamente $26{ }^{\circ} \mathrm{C}$ conforme solicitado pela NBR 12128 (ABNT, 2017c).

O que se verifica é que o tempo de pega dos gessos é curto e variável. Esse tempo de pega curto e variável, combinado com a ausência de critérios objetivos para a definição de quantidades de água de amassamento e procedimentos de controle de qualidade de recebimento (FREITAS, 2015; JOHN; CINCOTTO, 2007), tem levado a medidas de perdas de gesso de 30\% (AGOPYAN et al., 1998; SOUZA, 1998).

\section{Calor de hidratação}

Conforme Bardella (2011), o conhecimento do calor de hidratação e sua evolução em função do tempo são de interesse do ponto de vista científico e tecnológico. A curva obtida, temperatura x tempo, mostra a velocidade de hidratação do material, e, consequentemente, sua reatividade, sendo um importante parâmetro de controle da qualidade dos materiais (ANTUNES, 1999; BARDELLA, 2011).

No estudo, foram realizados ensaios de calorimetria térmica em pastas produzidas com todas as amostras coletadas e relação água/gesso dadas pela consistência normal. A curva de hidratação está apresentada na Figura 10. Entretanto, para valores precisos de tempo e temperatura de hidratação, deve-se utilizar calorimetria isotérmica.

As temperaturas máximas atingidas pelas pastas no final do período de hidratação foram similares: em torno de $55^{\circ} \mathrm{C}$.

Observa-se que todos os resultados apresentam um comportamento típico de curvas de calorimetria descrito por outros autores na literatura (ANTUNES; JOHN, 2000; BARDELLA, 2011; CLIFTON, 1973). As curvas de evolução de temperatura praticamente se sobrepõem em cada caso. Logo, como afirma Bardella (2011), os períodos de indução, nucleação e de endurecimento foram os mesmos quando comparados entre si. Além disso, isso é um indicativo de que a composição desses gessos também seja similar.

O início de pega pode ser identificado nas curvas de calorimetria, sendo o instante em que a taxa de elevação da temperatura ultrapassa $0,1{ }^{\circ} \mathrm{C} / \mathrm{min}$ (CLIFTON, 1973), e o de fim de pega é o ponto máximo de incremento da temperatura que corresponde à conclusão da hidratação (ANTUNES; JOHN, 2000).

Assim sendo, adotando-se essa metodologia para determinação dos tempos de pega, tem-se a comparação por calorimetria e aparelho de Vicat dada na Tabela 3.

É evidente que os tempos de pega obtidos pelos dois métodos são diferentes. Os resultados fornecidos pelo Aparelho de Vicat podem apresentar variações decorrentes do próprio mecanismo de realização do ensaio e também por influência do operador do equipamento (BARDELLA, 2011). 
Figura 10 - Curvas de hidratação de pastas de gessos produzidas com consistência normal

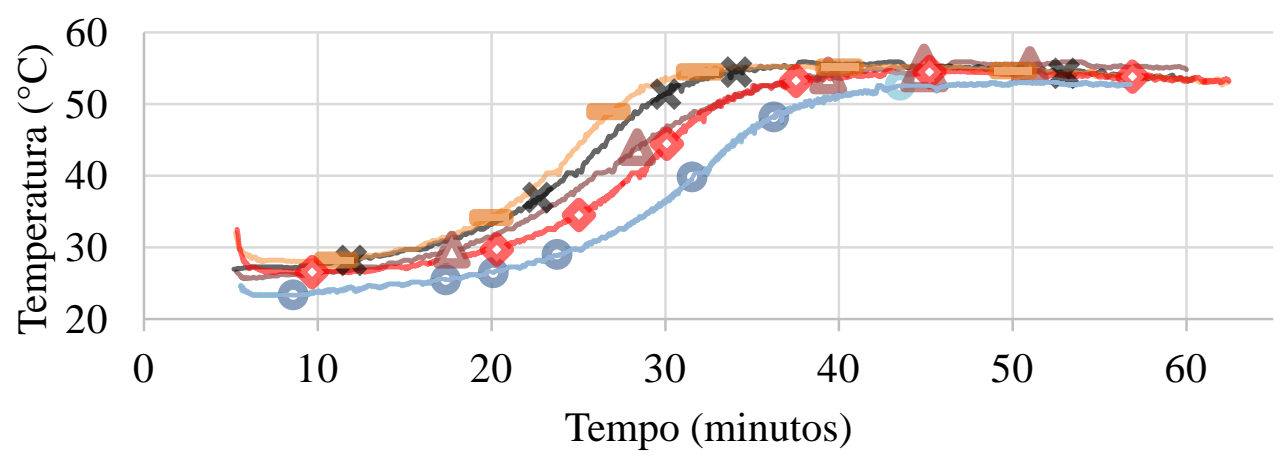

$\odot \mathrm{G} 1 \triangle \mathrm{G} 2 \times \mathrm{G} 3-\mathrm{G} 4 \odot \mathrm{G} 5$

Tabela 3 - Tempos de pega das pastas: calorimetria $\mathbf{x}$ aparelho de Vicat

\begin{tabular}{c|c|c|c|c}
\hline \multirow{2}{*}{ Gesso } & \multicolumn{2}{|c|}{ Início de pega (min) } & \multicolumn{2}{c}{ Fim de pega (min) } \\
\cline { 2 - 5 } & $\begin{array}{c}\text { Aparelho } \\
\text { de Vicat }\end{array}$ & Calorimetria1 $^{1}$ & $\begin{array}{c}\text { Aparelho } \\
\text { de Vicat }\end{array}$ & Calorimetria1 $^{1}$ \\
\hline G1 & 18,16 & 15,53 & 21,57 & 43,15 \\
G2 & 15,83 & 12,03 & 19,25 & 44,9 \\
G3 & 15,48 & 12,35 & 19,96 & 37,37 \\
G4 & 10,18 & 6,75 & 13,19 & 34,52 \\
G5 & 16,54 & 14,28 & 25,07 & 43,83 \\
\hline
\end{tabular}

Nota: ${ }^{1}$ os tempos determinados por calorimetria são estimativas.

Até o final do período de indução, não há elevação da temperatura e ainda não há di-hidrato formado. A consistência da pasta começa a ser modificada apenas no final desse período, ou seja, um pouco antes do início de pega dado por calorimetria (CLIFTON, 1973).

No início da pega determinado pela calorimetria, a quantidade de di-hidrato precipitado é aproximadamente igual a 0\% (CLIFTON, 1973; MAGNAN, $1973{ }^{2}$ apud ANTUNES, 1999), enquanto no início de pega determinado pelo aparelho de Vicat (ABNT, 2017c) aproximadamente $10 \%$ de di-hidrato está formado (STAV; BENTUR, $1995^{3}$ apud ANTUNES; JOHN, 2000), por isso, os tempos de pega pelos dois métodos resultam em valores distintos.

\section{Caracterização no estado endurecido}

\section{Dureza}

A NBR 12129 (ABNT, 2017d) utiliza para avaliação dessa propriedade pastas de gesso com a consistência normal, e não com relação a/g utilizada em revestimentos.

O ensaio de dureza (ABNT, 2017d) foi realizado com o método de penetração da esfera em corpos de prova cúbicos. A dureza não pode ser obtida, uma vez que a alta quantidade de gesso para certa quantidade de água (baixa relação água/gesso) levou à confecção de material excessivamente rígido com alta resistência mecânica e baixa resistência à deformação, que, durante o ensaio de penetração da esfera, resultou no rompimento dos corpos de prova.

De acordo com a NBR 13207 (ABNT, 2017e), o gesso para construção civil deve apresentar dureza maior ou igual a $20 \mathrm{~N} / \mathrm{mm}^{2}$.

\section{Resistência à compressão}

Os resultados de resistência à compressão das pastas de gessos produzidos estão apresentados na Figura 11.
2MAGNAN, M. Mecanisme et Cinétique de L'hydratation du Plâtre. Revue des Matériaux de Construction, v. 671, p. 28-31, 1973.

3STAV, E.; BENTUR, A. Characterization of the Hydration Process of Calcium Sulfate Hemihydrate by Simultaneous Evaluations of
Chemical and Physical Parameters. Advances in Cement Research, v. 7, n. 27, p. 113-116, 1995. 
Figura 11 - Resistência à compressão dos gessos com consistência normal

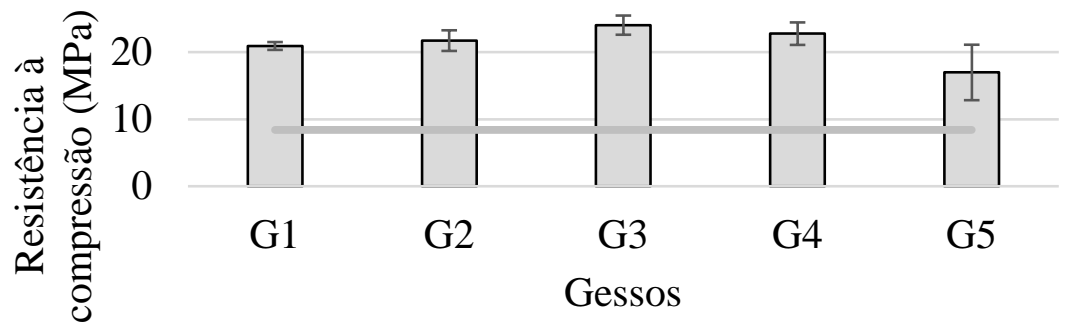

Resistência à compressão - consistência normal Mínimo NBR 13207 (ABNT, 1994)

Verifica-se que a resistência à compressão alcançada pelas pastas foi entre 16,97 MPa e 24,02 MPa. A NBR 13207 (ABNT, 2017e) não trata da avaliação da resistência à compressão do gesso para revestimento; entretanto, a NBR 12129 (ABNT, 2017d) aborda o método de ensaio para sua determinação.

A edição anterior da NBR 13207 (ABNT, 1994) recomendava uma resistência à compressão mínima de 8,40 MPa para um gesso de construção produzido com a relação água/gesso da consistência normal. A C28/C28M-10 (AMERICAN..., 2015), norma americana que trata das especificações dos gessos para revestimento, orienta que a resistência à compressão seja igual ou superior a 5,2 $\mathrm{MPa}$. Assim sendo, todas as amostras estão em conformidade com as duas normas.

Observa-se que a resistência à compressão apresenta diferenças entre si mesmo para aquelas produzidas com a mesma consistência. Esse comportamento é explicado pelas características específicas do material, além da possibilidade de variação inerente ao ensaio.

Karni e Karni (1995) afirmam que a resistência é resultado dos seguintes fatores: a qualidade do material (gesso e aditivos); a relação água/gesso; a idade do produto; e as condições de armazenamento do produto, também durante o endurecimento.

Se a relação for inferior a 0,6, há dificuldades com a trabalhabilidade. Para razões maiores que 0,6, tem-se um aumento da porosidade do produto final endurecido perdendo resistência mecânica (SILVA, 2010).

\section{Resistência de aderência à tração}

Os resultados de resistência de aderência à tração dos revestimentos produzidos e ensaiados conforme a NBR 13528 (ABNT, 2010) se encontram na Tabela 4. Embora as pastas de gessos tenham sido produzidas com a mesma relação água/gesso $(0,8)$ as suas trabalhabilidades foram distintas devido às diferenças dos materiais, como matéria-prima, finura e forma dos grãos.

Observa-se que a aderência dos revestimentos, independentemente do tratamento da base e do tipo de gesso, alcança o valor mínimo solicitado pela NBR 13207 (ABNT, 2017e) de 0,2 MPa. De acordo com os critérios da EN 13279-1 (BRITISH..., 2008), a resistência ao arrancamento do revestimento de gesso deve ser igual ou superior a 0,1 MPa.

As rupturas no ensaio se deram, majoritariamente, na interface substrato/revestimento, ou seja, foram rupturas de natureza adesiva e não coesiva.

O ensaio de aderência demonstrou grande variação dos resultados individuais dos corpos de prova. Entretanto, mesmo com essa variação, comentada por outros autores na bibliografia (COSTA; CARASEK, 2009; GONÇALVES, 2004), é o mais indicado para avaliação do desempenho de revestimentos. No geral, constatou-se também uma redução da resistência de aderência com o préumedecimento da base.

As características da base de aplicação do revestimento têm influência na sua resistência de aderência. Um substrato de maior permeabilidade proporciona maior penetração de produtos de hidratação, oferecendo a ancoragem do revestimento (CARASEK, 1996; COSTA, 2007; KAZMIERCZAK; BREZEZINSKI; COLLATTO, 2007; SOUSA; ALMEIDA; ALMEIDA, 2015).

Os blocos cerâmicos utilizados como base dos revestimentos das pastas de gesso, por serem de cerâmica vermelha obtida por queima, apresentam a face vidrada diminuindo a permeabilidade do substrato cerâmico e dificultando assim a microancoragem (BARDELLA, 2011). Além disso, o pré-umedecimento, indicado pela NBR 13867 (ABNT, 1997) - Revestimento interno de paredes e 
tetos com pasta de gesso - Materiais, preparo, aplicação e acabamento, reduz a capacidade de sucção da base, tendo como resultado uma redução do material que penetra nos poros da base que garante a ancoragem mecânica.

Entretanto, mesmo nessas situações, o revestimento apresentou aderência satisfatória. Os resultados de aderência do revestimento estão coerentes com os de Almeida (2014), Delgado e Pires Sobrinho (1997), Dias e Cincotto (1995) e Hincapie et al. (1997).

Foi realizada também uma avaliação para verificar se há diferença estatisticamente significativa entre os tipos de tratamento: sem pré-umedecimento e com pré-umedecimento da base. Os resultados mostram que, para a maioria dos resultados obtidos, há diferença entre os dois tipos de tratamento. Assim, recomenda-se que não haja o pré- umedecimento da base a fim de se garantir níveis maiores de aderência.

A partir de uma análise estatística, por meio do Teste de Tukey, pelo qual se faz confronto de amostra em pares para verificação de diferenças significativas, verifica-se que a maioria dos valores de água de cristalização e de resistência de aderência alcançados é semelhante entre si e não há diferenças estatisticamente significativas no grupo de resistência à compressão, o que é um indício de semelhança composicional de todos. Tais informações podem ser verificadas nas Tabelas $5 \mathrm{e}$ 6 , respectivamente. Ou seja, independentemente do porte da empresa e do processo de produção, as diferenças entre os gessos não foram significativas. Isso é um indicativo de que a matéria-prima do Polo Gesseiro do Araripe é de grande qualidade.

Tabela 4 - Resistência de aderência à tração dos revestimentos executados com e sem préumedecimento da base

\begin{tabular}{c|c|c}
\hline \multirow{2}{*}{ Gesso } & \multicolumn{2}{|c}{ Resistência de aderência (MPa) } \\
\cline { 2 - 3 } & $\begin{array}{c}\text { Sem pré- } \\
\text { umedecimento da base }\end{array}$ & $\begin{array}{c}\text { Com pré-umedecimento } \\
\text { da base }\end{array}$ \\
\hline G1 & $0,43 \pm 0,19$ & $0,25 \pm 0,07$ \\
G2 & $0,37 \pm 0,14$ & $0,23 \pm 0,09$ \\
G3 & $0,54 \pm 0,17$ & $0,34 \pm 0,12$ \\
G4 & $0,49 \pm 0,11$ & $0,25 \pm 0,10$ \\
G5 & $0,51 \pm 0,14$ & $0,39 \pm 0,10$ \\
\hline
\end{tabular}

Tabela 5 - Teste de Tukey para resultados de água de cristalização

\begin{tabular}{l|l|l|l|l}
\hline G2-G1 & G5-G1 & G4-G3 & G4-G2 & G3-G2* \\
\hline G3-G1 & G4-G1 & G5-G3 & G5-G2 & G5-G4 \\
\hline
\end{tabular}

Nota: *diferença estatisticamente significativa.

Tabela 6 - Teste de Tukey para resultados de resistência de aderência

\begin{tabular}{|c|c|c|c|c|}
\hline \multicolumn{5}{|c|}{$\begin{array}{c}\text { Avaliação da influência do tipo/fabricante de gesso entre os revestimentos } \\
\text { produzidos sem o pré-umedecimento da base. }\end{array}$} \\
\hline G2-G1 & G4-G1 & G4-G2 & G3-G2* & 'G4-G3 \\
\hline G3-G1 & G5-G1 & G5-G2 & G5-G4 & G5-G3 \\
\hline \multicolumn{5}{|c|}{$\begin{array}{l}\text { Avaliação da influência do tipo/fabricante de gesso entre os revestimentos } \\
\text { produzidos com o pré-umedecimento da base. }\end{array}$} \\
\hline G3-G1 & G4-G1 & G4-G3 & G4-G2 & G3-G2 \\
\hline G2-G1 & G5-G1 & G5-G3 & G5-G2* & G5-G4 \\
\hline \multicolumn{5}{|c|}{$\begin{array}{l}\text { Avaliação da existência de diferença entre o pré-umedecimento ou não da } \\
\text { base de revestimento de gesso de mesmo fabricante. }\end{array}$} \\
\hline G1s-G1c* & G2s-G2c* & G3s-G3c* & G4s-G4c* & G5s-G5c* \\
\hline
\end{tabular}

Nota: *diferença estatisticamente significativa.

Nota: Legenda:

$\mathrm{s}$ - sem pré-umedecimento; e

c - com pré-umedecimento. 


\section{Conclusão}

A partir do estudo, podem-se obter as seguintes conclusões:

(a) a matéria-prima (gipsita) e, consequentemente, o gesso produzido no Polo Gesseiro do Araripe, no Brasil, é de grande qualidade. Independentemente do porte da empresa, ou seja, do controle de produção, as propriedades dos gessos foram semelhantes;

(b) verificou-se também que os tempos de pega dos gessos brasileiros são curtos e variáveis;

(c) observa-se que a resistência de aderência dos revestimentos, independentemente do tratamento da base e do tipo de gesso, alcança o valor mínimo solicitado pela NBR 13207 (ABNT, 2017e) de 0,2 MPa, mesmo sendo aplicados em base cerâmica, que apresenta baixa rugosidade;

(d) houve redução da resistência de aderência com o pré-umedecimento da base, provavelmente pela diminuição de sua capacidade de sucção, tendo como resultado uma redução do material que penetra nos poros que garantiria a ancoragem mecânica. Entretanto, mesmo nessas situações, o revestimento apresentou aderência satisfatória; e

(e) não há diferenças estatisticamente significativas de resistência de aderência entre os gessos para a maioria dos valores encontrados, 0 que mostra o desempenho semelhante de todos eles.

\section{Referências}

ADRIEN, J. et al. In-Situ X-Ray Tomographic Monitoring of Gypsum Plaster Setting. Cement and Concrete Research, v. 82, p. 107-116, 2016.

AGOPYAN, V. et al. Alternativas Para Redução de Desperdício de Materiais nos Canteiros de Obras. 1998. v. 2, p. 224-249. Coletânea Habitare, Inovação, Gestão da Qualidade \& Produtividade e Disseminação do Conhecimento na Construção Habitacional.

ALMEIDA, Y. K. R. Estudo de Resistência de Aderência de Revestimento Executados com Pasta de Gesso, Considerando Diferentes Substratos. 75 f. 2014. Monografia (Trabalho de Conclusão de Curso) - Universidade Federal Vale do São Francisco, 2014.

\footnotetext{
AMERICAN SOCIETY FOR TESTING AND MATERIALS. C28/C28M-10: standard specification for gypsum plasters. Pennsylvania, 2015.
}

ANTUNES, R. P. N. Estudo da Influência da Cal Hidratada nas Pastas de Gesso. 134 f. São Paulo, 1999. Dissertação (Mestrado em Engenharia Civil) - Escola Politécnica, Universidade de São Paulo, São Paulo, 1999.

ANTUNES, R. P. N.; JOHN, V. M. O Conceito de Tempo Útil das Pastas de Gesso. São Paulo: EPUSP, 2000. Boletim Técnico PCC n. 254.

ASSOCIAÇÃO BRASILEIRA DE NORMAS TÉCNICAS. NBR 12127: gesso para construção: determinação das propriedades físicas do pó. Rio de Janeiro, 2017a.

\section{ASSOCIAÇÃO BRASILEIRA DE NORMAS} TÉCNICAS. NBR 12128: gesso para construção: determinação das propriedades físicas da pasta. Rio de Janeiro, 2017c.

\section{ASSOCIAÇÃO BRASILEIRA DE NORMAS}

TÉCNICAS. NBR 12129: gesso para construção: determinação das propriedades mecânicas. Rio de Janeiro, 2017d.

\section{ASSOCIAÇÃO BRASILEIRA DE NORMAS}

TÉCNICAS. NBR 12130: gesso para construção: determinação da água livre e de cristalização e teores de óxido de cálcio e anidrido sulfúrico. Rio de Janeiro, 2017b.

\section{ASSOCIAÇÃO BRASILEIRA DE NORMAS TÉCNICAS. NBR 13207: gesso para construção civil: requisitos. Rio de Janeiro, 1994.}

\section{ASSOCIAÇÃO BRASILEIRA DE NORMAS} TÉCNICAS. NBR 13207: gesso para construção civil: requisitos. Rio de Janeiro, 2017e.

\section{ASSOCIAÇÃO BRASILEIRA DE NORMAS}

TÉCNICAS. NBR 13528: revestimento de paredes de argamassas inorgânicas: determinação da resistência de aderência à tração. Rio de Janeiro, 2010.

\section{ASSOCIAÇÃO BRASILEIRA DE NORMAS}

TÉCNICAS. NBR 13867: revestimento interno de paredes e tetos com pasta de gesso: materiais, preparo, aplicação e acabamento. Rio de Janeiro, 1997.

\section{ASSOCIAÇÃO MERCOSUL DE}

NORMALIZAÇÃO. NM 23: cimento portland e outros materiais em pó: determinação da massa específica. São Paulo, 2000.

BARBOSA, A. A.; FERRAZ, A. V.; SANTOS, G. A. Caracterização Química, Mecânica e Morfológica do Gesso Obtido do Polo do Araripe. Cerâmica, v. 60, p. 501-508, 2014. 
BARDELLA, P. S. Análise das Propriedades de Pastas de Gesso de Construção Reciclado. $235 \mathrm{f}$. Campinas, 2011. (Tese) - Doutorado em Engenharia Civil, Universidade Estadual de Campinas, Campinas, 2011.

BRITISH STANDARD EUROPEAN NORM. EN 13279-1: gypsum binders and gypsum plasters: definitions and requirements. Bruxelas, 2008.

CAMARINI, G.; PIMENTEL, L. L.; SÁ, N. H. R. Assessment of the Material Loss in Walls Renderings with B-Hemihydrate Paste. Applied Mechanics and Materials, v. 71/78, p. 1.2421.245, 2011.

CAMARINI, G. et al. Effect of Citric Acid on Properties of Recycled Gypsum Plaster to Building Components. Construction and Building Materials, v. 124, p. 383-390, 2016.

CARASEK, H. Aderência de Argamassas à Base de Cimento Portland a Substratos Porosos: avaliação dos fatores intervenientes e contribuição ao estudo do mecanismo da ligação. São Paulo, 1996. Tese (Doutorado em Engenharia Civil) Escola de Engenharia, Universidade de São Paulo, São Paulo, 1996.

CARDOSO, F. A. et al. Squeeze Flow as a Tool for Developing Optimized Gypsum Plasters.

Construction and Building Materials, v. 23, p. 1.349-1.353, 2009.

CARNEIRO, A. M. P. Contribuição ao Estudo da Influência do Agregado nas Propriedades de Argamassas Compostas a Partir de Curvas Granulométricas. São Paulo, 1999. 170 f. Tese (Doutorado em Engenharia Civil) - Escola Politécnica da Universidade de São Paulo, São Paulo, 1999.

CAUFIN, B.; PAPO, A. Rheological Testing Methods for Gypsum Plaster Pastes. RILEM Materials and Structures, v. 95, p. 315-321, 1983.

CINCOTTO, M. A.; AGOPYAN, V.; FLORINDO, M. C. O Gesso Como Material de Construção: propriedades físicas e mecânicas, $2^{\mathrm{a}}$ parte. In: INSTITUTO DE PESQUISAS TECNOLÓGICAS. Tecnologia das Edificações. São Paulo, 1988.

CLIFTON, J. R. Some Aspects of the Setting and Hardening of Gypsum Plaster. NBS Technical Note, v. 755, 1973.

COSTA, E. B. C.; CARASEK, H. Influência dos Parâmetros de Ensaio na Determinação da Resistência de Aderência de Revestimentos de Argamassa. Ambiente Construído, Porto Alegre, v. 9, n. 4, p. 17-35, out./dez. 2009.
COSTA, E. Investigação do Método de Ensaio de Determinação da Resistência de Aderência de Revestimentos de Argamassa. 205 f. Goiânia, 2007. Dissertação (Mestrado) - Curso de Mestrado em Engenharia Civil, Universidade Federal de Goiás, Goiânia, 2007.

COSTA, J. E. B. Análise Comparativa Entre as Propriedades do Gesso Obtido de Rejeito da Produção de Sal e Gessos Comerciais. 79 f. Natal, 2013. Dissertação (Mestrado em Engenharia Civil) - Escola de Engenharia, Universidade Federal do Rio Grande do Norte, Natal, 2013.

DE MILITO, J. A. Avaliação do Comportamento de Pastas de Gesso com Cimento Portland e Sílica Ativa Para Revestimento. $228 \mathrm{f}$. Campinas, 2001. Dissertação (Mestrado em Engenharia Civil) - Faculdade de Engenharia Civil, Universidade Estadual de Campinas, Campinas, 2001.

DELGADO, C. B.; PIRES SOBRINHO, C. W. A. Revestimentos de Gesso (Pasta e Argamassa) Determinação das Propriedades Mecânicas. In: SIMPÓSIO BRASILEIRO DE TECNOLOGIA DAS ARGAMASSAS, 2., Salvador, 1997. Anais... Salvador, 1997.

DIAS, A. M. N.; CINCOTTO, M. A.

Revestimento à Base de Gesso de Construção. São Paulo: EPUSP, 1995. Boletim Técnico PCC n. 142.

FREITAS, C. de. Materiais de Construção. Rede Internacional de Universidades Laureate. São Paulo, 2015. Disponível em:

$<$ https://issuu.com/eadunifacs/docs/materiais_cons trucao >. Acesso em: 15 jan. 2018.

GARTNER, E. M. Cohesion and Expansion in Polycrystalline Solids Formed by Hydration Reactions: the case of gypsum plasters. Cement and Concrete Research, v. 39, p. 289-295, 2009.

GONÇALVES, S. R. C. Variabilidade e Fatores de Dispersão da Resistência de Aderência nos Revestimentos em Argamassa: estudo de caso. 167 f. Brasília, 2004. Dissertação (Mestrado em Engenharia Civil) - Escola de Engenharia, Universidade de Brasília, Brasília, 2004.

HINCAPIE, H. et al. Revestimento de Gesso LisoAvaliação da Aderência e Dureza Superficial, Sobre Diversos Substratos. In: GYPSUM FAIR, Olinda, 1997. Anais... Olinda, 1997.

JOHN, V. M.; ANTUNES, R. P. N. Argamassas de Gesso. Ambiente Construído, Porto Alegre, v. v2, n. 1, p. 29-38, 2002.

JOHN, V. M.; CINCOTTO, M. A. Gesso de Construção Civil. In: ISAIA, G. C. Materiais de Construção Civil. São Paulo: IBRACON, 2007. 
KARNI, J.; KARNI, E. Gypsum in Construction: origin and properties. Materials and Structures, v. 28, n. 176, p. 92-100, mar. 1995.

KAZMIERCZAK, C. S.; BREZEZINSKI, D. E.; COLLATTO, D. Influência das Características da Base na Resistência de Aderência à Tração e na Distribuição de Poros de Uma Argamassa.

Estudos Tecnológicos, v. 3, n. 1, p. 47-58, jan./jun. 2007.

LEWRY, A. J.; WILLIAMSON, J. The Setting of Gypsum plaster: part III: the effect of additives and impurities. Journal of Material Science, v. 29, p. 6.085-6.090, 1994.

PINHEIRO, S. M. M. Gesso Reciclado: avaliação das propriedades para uso em componentes. $330 \mathrm{f}$. São Paulo, 2011. Tese (Doutorado em Engenharia Civil) - Escola de Engenharia, Universidade Estadual de Campinas, São Paulo, 2011.

SCHMITZ, I. B. T. A.; TAVARES, Y. V. P. Estudo do Desempenho da Pasta de Gesso com Adição de Seu Resíduo Como Revestimento Interno de Paredes. In: SIMPÓSIO BRASILEIRO DE TECNOLOGIA DAS ARGAMASSAS, 8., Curitiba, 2009. Anais... Curitiba, 2009.

SILVA, M. G. S. Desenvolvimento de Compósitos à Base de Gesso e Pó de Fibras de Coco. 84 f. São Cristovão, 2010. Dissertação (Mestrado em Engenharia Civil) - Escola de Engenharia, Universidade Federal de Sergipe, São Cristovão, 2010.
SOUSA, J. G. G.; ALMEIDA, Y. K. R.; ALMEIDA, M. O. Estudo da Resistência de Aderência de Revestimentos Executados Com Pasta de Gesso em Diferentes Substratos. In: SIMPÓSIO BRASILEIRO DE TECNOLOGIA DAS ARGAMASSAS, 11., Porto Alegre, 2015. Anais... Porto Alegre, 2015.

SOUZA, U. E. L. Alternativas Para a Redução de Desperdício de Materiais nos Canteiros de Obras, Relatório FINEP. 1998, v. 5, p. 184-189.

TYDLITÁT, V.; MEDVED, I.; CERNÝ, R. Determination of a Partial Phase Composition in Calcined Gypsum by Calorimetric Analysis of Hydration Kinetics. Journal of Thermal Analysis and Calorimetry, v. 109, p. 57-62, 2012.

YE, Q. et al. Effect of Particle Size Distribution on the Hydration and Compressive Strength Development of $\alpha$-Calcium Sulfate Hemihydrate Paste. Powder Technology, v. 207, p. 208-214, 2011.

YU, Q. L.; BROUWERS, H. J. H. Thermal Properties and Microstructure of Gypsum Board and Its Dehydration Products: a theoretical and experimental investigation. Fire and Materials, v. 36, p. 575-589, 2012.

\section{Agradecimentos}

À Capes, pelo apoio financeiro.

\begin{abstract}
Fernanda Cavalcanti Ferreira
Departamento de Engenharia Civil, Centro de Tecnologia e Geociências | Universidade Federal de Pernambuco | Av. da Arquitetura, Cidade Universitária | Recife - PE - Brasil | CEP 50740-550 | Tel.: (81) 2126-8977 | E-mail: fernandacavalcantif@gmail.com

J osé getulio Gomes de Sousa

Colegiado de Engenharia Civil | ${ }^{2}$ Fundação Universidade Federal do Vale do São Francisco | Av. Antônio Carlos Magalhães, 510, Santo Antônio | J uazeiro - BA - Brasil | Caixa Postal 309 | CEP 48902-300 | Tel.: (74) 2102-7624 | E-mail: jose.getulio@univasf.edu.br
\end{abstract}

\title{
Arnaldo Manoel Pereira Carneiro
}

Centro de Tecnologia e Geociências | ${ }^{3}$ Universidade Federal de Pernambuco | Rua Acadêmico Hélio Ramos, s/n, Cidade Universitária | Recife - PE - Brasil | CEP 50740-530 | Tel.: (81) 2126-8220 Ramal 35 | E-mail: ampc@ufpe.br

Revista Ambiente Construído

Associação Nacional de Tecnologia do Ambiente Construído

Av. Osvaldo Aranha, 99 - 3o andar, Centro

Porto Alegre - RS - Brasil CEP 90035-190

Telefone: +55 (51) 3308-4084 Fax: +55 (51) 3308-4054

www. seer. ufrgs. br/ ambienteconstruido

E-mail: ambienteconstruido@ufrgs.br 